\title{
Stress Ocupacional em Profissionais de Segurança Pública: Um Estudo com Militares da Guarda Nacional Republicana
}

\author{
Occupational Stress in Professionals of Public Security: A Study \\ with Military Agents of the Republican National Guard
}

\author{
Jorge M. P. Afonso \& A. Rui Gomes* \\ Universidade do Minho
}

\begin{abstract}
Resumo
Este trabalho analisa o "stress" ocupacional em 95 militares da Guarda Nacional Republicana Portuguesa. Para tal, utilizámos um protocolo de avaliação com medidas do "stress" global, "burnout", "coping” proactivo, comprometimento organizacional, satisfação com a vida e satisfação/realização profissional. Os indicadores de fidelidade e validade dos instrumentos foram muito aceitáveis. Os resultados de "burnout" por dimensão apontaram níveis apreciáveis de exaustão emocional (12\%), seguidos do cinismo (10\%) e da baixa eficácia profissional (8\%) (mas nenhum participante registou valores de "burnout" nas três dimensões, em simultâneo), observando-se também variáveis distintas na predição destas três áreas. A análise discriminante entre subgrupos da amostra permitiu verificar dois aspectos principais: (a) menores níveis de comprometimento organizacional e maior utilização de "coping" proactivo nos militares mais novos e/ou inexperientes e (b) maior desejo de abandonar o emprego/trabalho bem como maior cinismo parecem identificar os profissionais mais velhos e/ ou mais experientes. No final, os autores discutem a importância de a investigação futura analisar os efeitos da maior tendência para o cinismo com o aumento da idade dos participantes e os efeitos do menor comprometimento organizacional por parte dos mais novos em termos da sua eficácia profissional.

Palavras-chave: Stress ocupacional; Burnout; Coping; Comprometimento organizacional; Satisfação.
\end{abstract}

\begin{abstract}
This work analyzes occupational stress in 95 Portuguese military agents of the Republican National Guard. We applied an evaluation protocol with measures of global stress, burnout, proactive coping, organizational commitment, satisfaction with life, and professional satisfaction/accomplishment. The fidelity and validity results of the instruments were very acceptable. The results of burnout pointed out a remarkable level of emotional exhaustion (12\%) followed by cynicism (10\%) and low professional effectiveness (8\%) (but we have not found any participant with burnout values in the three dimensions simultaneously), and different variables were observed in the prediction of those three areas. The discriminant analysis among subgroups allowed us to verify two main aspects: (a) lower levels of organizational commitment and more use of proactive coping in younger and/or inexperienced military agents and (b) higher desire to leave the job/profession and cynicism in older and/or more experienced professionals. At the end, the authors discuss the importance of future research to analyze the effects of a higher tendency to use cynicism as their age increase and the consequences of a lower organizational commitment in terms of professional efficacy of the younger agents. Keywords: Occupational stress, Burnout; Coping; Organizational commitment; Satisfaction.
\end{abstract}

A actividade profissional das forças de segurança é considerada uma das ocupações mais stressantes do mundo (Dantzer, 1987; Selye, 1984). Os motivos para esta classificação prendem-se com a natureza das funções prestadas

* Endereço para correspondência: Universidade do Minho, Instituto de Educação e Psicologia, Campus de Gualtar, Braga, Portugal, 4710-057.E-mail: rgomes@ iep.uminho.pt Os autores gostariam de agradecer o apoio do Comando da Guarda Nacional Republicana (Grupo Territorial de Braga) para a realização deste trabalho, sem o qual teria sido impossível concretizar a investigação. Este reconhecimento é igualmente extensível aos Comandantes de Posto dos vários locais onde foram recolhidos os dados bem como a todos os participantes que de, de forma motivada e amável, se ofereceram para participar no estudo.

Os autores agradecem as valiosas correcções e sugestões fornecidas pelos revisores anónimos designados pelo Conselho Editorial da Revista Psicologia: Reflexão e Crítica, que ajudaram a melhorar e enriquecer a versão final deste artigo. onde, para além dos problemas mais comuns a outras ocupações (ex: trabalho por turnos, excesso de horas de trabalho, etc.), estes profissionais têm ainda de enfrentar uma área de pressão específica a esta actividade: o risco de vida para o próprio e para os outros.

A exposição a estas potenciais fontes de tensão tem sido associada a múltiplos problemas, desde a componente mais física (ex: doenças cardiovasculares, elevado colesterol, problemas de estômago, etc.), até uma dimensão mais psicológica, relacionada vulgarmente a desordens mentais e ao "stress" pós-traumático (Abdollahi, 2002; Carlier, Lamberts, \& Gersons, 2000; Stephens, Long, \& Flett, 1999; Violanti et al., 2006).

Apesar da importância do tema do "stress" ocupacional em forças policiais, tanto para o indivíduo como para a 
sociedade em geral, a verdade é que esta classe profissional não se tem assumido como um grupo particularmente estudado. A título meramente ilustrativo, Shaufeli e Enzmann (1998) consideram surpreendente o facto de existirem tão poucas investigações centradas no "burnout" (esgotamento) em polícias, uma vez que este fenómeno representa um dos factores psicológicos mais associado aos problemas no trabalho. Esta situação é ainda mais evidente em Portugal, escasseando os estudos e, consequentemente, o conhecimento sobre as características e exigências desta ocupação e o modo como estes profissionais percepcionam e gerem as exigências do trabalho.

É neste sentido que desenvolvemos esta investigação, observando a experiência de "stress" ocupacional numa das principais forças policiais portuguesas: a Guarda Nacional Republicana (GNR). Ao longo deste estudo faremos referência a estes profissionais como sendo "militares", uma vez que, organicamente, esta força de segurança é constituída por militares organizados num corpo especial de tropas com a missão da manutenção da ordem pública e a segurança de pessoas e bens.

A estrutura metodológica seguida neste estudo procurou incluir algumas dimensões de carácter individual e outras de cariz mais ocupacional e organizacional, seguindo-se assim as orientações fornecidas por Hart, Wearing e Headey (1995) ao proporem estes três domínios como sendo os mais adequados para ajudar a compreender o problema do "stress" profissional nos polícias. Neste sentido, assumimos neste trabalho a necessidade de estudar a relação entre o "stress" e um conjunto de dimensões mais psicológicas (e.g., nível de "stress", "burnout", "coping” ou confronto psicológico e satisfação com a vida), a que se seguem outras de natureza tipicamente ocupacional (e.g., satisfação e realização profissional) e, por último, outras de cariz mais organizacional (e.g., comprometimento relativamente ao local de trabalho). Ao optarmos por este leque distinto de variáveis, procurámos alargar o mais possível a recolha de informação e, consequentemente, o entendimento deste fenómeno.

Assim sendo, o trabalho levado a cabo pretendeu atingir os seguintes objectivos: (a) Examinar a frequência e prevalência de variáveis psicológicas, ocupacionais e organizacionais associadas ao exercício profissional dos militares (e.g., nível global de "stress", "burnout", "coping", satisfação com a vida, satisfação e realização profissional e comprometimento organizacional); (b) Analisar as relações e associações entre as variáveis em estudo; (c) Observar as variáveis preditoras da experiência de "burnout" e (d) Identificar os factores que melhor diferenciam (ou "discriminam") os militares, em função de um conjunto de características demográficos e socioprofissionais.

\section{Método}

\section{Amostra}

Participaram neste estudo 95 militares da Guarda Nacional Republicana (GNR), a trabalhar em diferentes locais na região norte de Portugal, sendo 90 do sexo masculino
(94.7\%) e 5 do sexo feminino (5.3\%). As idades variaram entre os 22 e os 54 anos, com uma média de 37 anos. O estado civil colocou 30 profissionais na condição de solteiros (31.9\%), 60 como casados $(63.8 \%)$ e quatro como divorciados $(4.3 \%)$ (um dos participantes não forneceu informações a este respeito). Em termos de habilitações escolares, o ensino secundário foi o grau académico mais frequente $(n=49 ; 51.6 \%)$, seguindo-se o $2^{\circ}$ ciclo $(n=21$; $22.1 \%)$, o $1^{\circ}$ ciclo $(n=15 ; 15.8 \%)$, o $3^{\circ}$ ciclo $(n=9 ; 9.5 \%)$ e, por último, o ensino superior $(n=1 ; 1.1 \%)$. Do ponto de vista profissional, 62 participantes eram soldados (68.1\%), 20 eram cabos (22\%) e nove eram sargentos (9.9\%) (quatro participantes não forneceram informações a este respeito). Os anos de prática profissional variaram entre um e 27 anos, com uma média de pouco mais de oito anos. O número de horas de trabalho semanal apresentou uma grande amplitude de tempo, desde o tempo mínimo de 25 horas até um máximo de 70 horas (média de 48 horas semanais).

\section{Instrumentos e Medidas}

Foi administrado a todos os profissionais um conjunto de instrumentos destinados a obter informações acerca das variáveis em análise neste estudo.

Questionário Demográfico. Este instrumento, para além de obter informações acerca do sexo, idade, estado civil e número de filhos, recolheu dados relativamente à formação académica bem como às características e condições gerais de trabalho (e.g., contexto de acção, posição profissional ocupada, funções desempenhadas, experiência profissional e horas de trabalho por semana).

Escala de Nível Global de "Stress" (ENGS). Trata-se de uma medida desenvolvida a partir dos trabalhos originais de Kyriacou e Sutcliffe (1978). O instrumento é representado por um único item, avaliando a percepção dos militares face ao nível geral de "stress" que sentem na sua actividade profissional (Gomes, 1998; Gomes, Melo, \& Cruz, 2000). A escala de resposta é de cinco pontos, desde o zero (Nenhum "stress") até ao quatro (Elevado "stress"), dando-nos indicações acerca do modo como o profissional percepciona a sua actividade laboral.

Inventário de "Burnout" de Maslach - Versão Geral (IBM-VG). Trata-se da versão traduzida e adaptada por Gomes (2007) a partir dos trabalhos originais de Maslach, Jackson e Leiter (1996) e Schaufeli, Leiter, Maslach e Jackson (1996). Este instrumento foi desenvolvido com o objectivo de avaliar os níveis de "burnout" (esgotamento) evidenciados por trabalhadores não incluídos nas tradicionais profissões de ajuda da área da saúde. Assim sendo, o IBM-VG é um instrumento de auto-registo acerca dos sentimentos relacionados com o trabalho, distribuindo-se por três dimensões: (a) exaustão emocional: analisa os sentimentos de sobrecarga e exaustão emocional devido às exigências do trabalho (ex: sensação de esgotamento com o trabalho); (b) cinismo: pretende medir as respostas de indiferença e as atitudes de distanciamento relativamente ao trabalho (ex: dúvidas acerca da importância e interesse da profissão) e (c) eficácia profissional: usada para avaliar 
as expectativas de eficácia dos profissionais relativamente ao trabalho (ex: sentimentos positivos acerca da capacidade pessoal para resolver os problemas). O inventário é constituído por 16 afirmações, distribuídas pelas três subescalas referidas, sendo os itens respondidos numa escala tipo "Likert" de sete pontos $(0=$ Nunca; $6=$ Todos os dias). A pontuação é obtida através da soma dos itens de cada dimensão, dividindo-se depois o valor encontrado pelo número de itens que constituem cada subescala. Assim, os resultados totais podem variar entre um mínimo de zero e um máximo de seis. O valor máximo reflecte elevados índices de exaustão emocional, despersonalização e eficácia profissional. A interpretação dos resultados deve ser efectuada de um modo parcial, em cada subescala, sendo estas consideradas isoladamente e não através de uma combinação dos valores num único valor/factor global (Maslach et al., 1996). Neste sentido, elevados níveis de "burnout" estão associados a elevados "scores" de exaustão emocional e despersonalização, mas também a baixos "scores" de eficácia profissional. Mais à frente neste trabalho, apresentamos os critérios de cálculo dos valores de "burnout" em cada uma das três dimensões.

Escala de "Coping” Proactivo (ECP). Este instrumento é uma das subescalas incluídas no "Inventário de Coping Proactivo" desenvolvido por Greenglass, Schwarzer e Taubert (1999) que engloba sete dimensões distintas. A tradução e adaptação efectuadas por Cruz e Gomes (2007) refere-se apenas à escala de "coping" proactivo, que tem por objectivo avaliar as estratégias de confronto "activas" utilizadas pelas pessoas quando se confrontam com um determinado problema (ex: imaginar as soluções a adoptar, percepcionar os obstáculos como um desafio, etc.). $\mathrm{O}$ instrumento compreende uma subescala, num total de 14 itens respondidos numa escala tipo "Likert" de quatro pontos (1=Nada verdadeiro; 4=Completamento verdadeiro). A pontuação é obtida através da soma e posterior divisão dos itens da subescala, devendo-se atender aos que são formulados pela negativa e que devem ser invertidos na cotação final. Valores mais elevados significam maior utilização do confronto proactivo.

Escala de Comprometimento Organizacional (ECO). Este instrumento foi traduzido e adaptado por Gomes (2006) a partir dos trabalhos originais de Mowday, Steers e Porter (1979). A escala visa avaliar os sentimentos, atitudes e valores positivos assumidos pelos profissionais relativamente ao seu local de trabalho (ex: sentimento de orgulho por fazer parte da organização em causa, motivação e disponibilidade para fazer sacrifícios pessoais em nome da organização, etc.). A versão original é constituída por quinze itens sendo, no entanto, possível utilizar apenas nove itens de modo a extrair o factor original avaliado pelo instrumento (ver Mowday, Porter, \& Steers, 1982). Os itens são respondidos numa escala tipo "Likert" de cinco pontos ( $1=$ Discordo totalmente; $5=$ Concordo totalmente), extraindo-se um "score" total resultante da soma das pontuações obtidas, dividindo-se depois o valor encontrado pelo número de itens da escala.
Escala de Satisfação com a Vida (SWLS). Este instrumento foi traduzido e adaptado por Neto $(1993,1999)$ a partir dos trabalhos originais de Diener, Emmons, Larsen e Griffin (1985). A escala tem por objectivo avaliar a satisfação com a vida enquanto processo cognitivo (julgamento pessoal acerca da vida), assumindo-se que é mais relevante solicitar à pessoa uma análise global da sua vida do que efectuar uma avaliação da satisfação em domínios específicos da vida. $\mathrm{O}$ instrumento é constituído por cinco itens, apresentados num formato tipo "Likert" de cinco pontos (1=Discordo muito; 5=Concordo muito), solicitando-se às pessoas que avaliem a sua vida em geral (ex: as minhas condições de vida são excelentes). A pontuação final é calculada através da soma dos valores obtidos em cada um dos cinco itens;

Escala de Satisfação e Realização (ESR): Este instrumento foi desenvolvido com o objectivo de avaliar os níveis de satisfação e realização profissional (Gomes, 1998; Gomes et al., 2000) e teve por base instrumentos similares utilizados por Boice e Myers (1987), Rodolfa e Kraft (1988) e Thoresen, Miller e Krauskopf (1989). A escala é composta por cinco questões acerca da carreira e satisfação profissional, nomeadamente, (a) vontade em optar pela mesma via de formação se os militares tivessem uma nova oportunidade de escolher uma saída profissional (respostas numa escala dicotómica de "sim" e "não"); (b) nível de satisfação profissional actual; (c) nível de realização profissional actual; (d) desejo de abandonar o actual local de trabalho durante os próximos cinco anos; e (e) desejo de abandonar a profissão durante os próximos cinco anos. Estas últimas quatro questões foram apresentadas numa escala tipo "Likert" de seis pontos (1=Muito Baixo; 6=Muito Alto), significando os valores mais elevados nos diferentes itens maior satisfação e realização profissional bem como um maior desejo de abandonar o emprego e a profissão.

\section{Procedimento}

A investigação iniciou-se com o pedido de autorização ao Comando do Grupo Territorial de um distrito da zona norte de Portugal, explicando-se os objectivos do estudo e os procedimentos a implementar na recolha, tratamento e divulgação dos dados. Após a anuência do Comando, iniciamos o trabalho de campo propriamente dito, contactando os Comandantes de Posto de cada local de trabalho. Combinado o momento para a recolha dos dados, distribuiu-se o protocolo de avaliação que incluía, anexa, uma carta de apresentação, dirigida aos participantes, acerca dos objectivos e implicações da investigação, assegurando-se o carácter voluntário da participação. De salientar que a recolha dos questionários foi sempre efectuada no local de trabalho dos profissionais, em dias de instrução de modo a não interferir no normal funcionamento da actividade profissional, organizando-se um local reservado para o preenchimento do protocolo de avaliação. No sentido de garantir a confidencialidade e anonimato dos dados recolhidos, foi fornecido a todos os inquiridos um envelope no qual introduziam o questionário devidamente fechado. A 
abertura dos envelopes contendo os questionários recebidos, foi sempre da única e exclusiva responsabilidade do grupo de investigadores deste estudo, de forma a garantir a confidencialidade dos dados. Assim sendo, o protocolo que englobava os instrumentos atrás referidos foi distribuído junto de 95 militares, tendo sido recebidos e considerados válidos para efeitos do presente estudo todos os questionários entregues.

\section{Resultados}

O tratamento e análise dos dados foram efectuados no programa SPSS (versão 15.0 para Windows) e incluiu vários procedimentos que serão explicados ao longo desta parte do trabalho.

\section{Estrutura Factorial dos Instrumentos e Consistência Interna das Dimensões}

No sentido de testar a estrutura factorial dos instrumentos referidos, efectuámos uma análise factorial das componentes principais, sem pré-definição do número de factores, através da rotação ortogonal (procedimento "varimax"), com normalização de Kaiser ("eigenvalue" igual ou superior a 1). Para a retenção dos itens em cada uma das escalas, foram fixados os seguintes critérios: (a) saturação igual ou superior a .40 de cada item no factor hipotético e apenas num único factor; (b) aceitação da solução factorial final a partir do momento em que explicasse pelo menos $50 \%$ da variância total; (c) existência de uma coerência entre a solução factorial e os itens que constituem cada um dos factores e (d) cada factor deveria ser representado, pelo menos, por dois itens (ver Gorsuch, 1983; Hair, Anderson, Tatham, \& Black, 1995).

Começando pelo IBM-VG, foram identificados os três factores originais da escala que explicaram $62.1 \%$ de variância total, incluindo 15 itens distribuídos da seguinte forma: exaustão emocional, com cinco itens e um valor de "Alpha" de .86; eficácia profissional com seis itens e um valor de "Alpha" de .85 e cinismo com quatro itens e uma "Alpha" de .64. A este nível, é apenas de salientar o valor de "alpha" obtido nesta última subescala como estando abaixo do valor de.70 recomendado por Nunnally (1978), mas tal resultado reflecte as mesmas dificuldades enunciadas pelos autores do instrumento (Maslach et al., 1996) bem como por outros autores (ver Leiter \& Durup, 1996). Neste sentido, optamos por manter a subescala nas análises subsequentes deste estudo alertando-se, no entanto, para o valor verificado neste estudo.

A escala de "coping" proactivo reflectiu a sua natureza unidimensional, com um factor explicativo de $42.4 \%$ da variância e um valor de "alpha" de .83 para um total de nove itens. A este nível, é de referir que o valor de percentagem de variância explicada pelo factor situa-se abaixo dos $50 \%$ estabelecidos para este estudo mas resolvemos manter a dimensão nas análises seguintes, uma vez que a mesma reflecte perfeitamente o constructo original apresentado pelos autores (ver Greenglass et al., 1999).
No que se refere ao comprometimento organizacional, também observamos o carácter unidimensional do instrumento com um factor a explicar $53.5 \%$ da variância e um valor de "alpha" de .85 para um total de oito itens.

Neste mesmo sentido, a escala de satisfação com a vida evidenciou um único factor para os cinco itens (tal como previsto), agregando $59.1 \%$ da variância ("alpha" de .82).

Finalmente, a escala de satisfação e realização profissional permitiu a divisão em dois grandes factores $(79.8 \%$ de variância explicada), nomeadamente a vontade dos profissionais abandonarem o local de trabalho e a profissão ("alpha" de .71) e a satisfação e realização profissional (“alpha” de .75).

\section{Estatísticas Descritivas e Associações entre as Variáveis}

Começando pelo "nível global de stress" que os militares sentem geralmente no exercício da sua actividade profissional, é de realçar o facto de 54\% dos participantes terem relatado índices globais de "stress" muito significativos (junção dos valores "bastante" e "elevado" da escala "Likert").

Relativamente aos níveis de esgotamento, utilizámos as indicações sugeridas por Shirom (1989) para calcular os valores problemáticos em cada uma das três facetas do "burnout". Neste caso, o autor propõe a utilização dos valores da escala "Likert" como referência para estabelecer os "pontos" de corte das três escalas, em vez dos valores normativos sugeridos pelos autores da escala (Maslach et al., 1996). Neste último caso, a identificação do perfil de "burnout" dá-se pelo cálculo das pontuações no terço superior da distribuição dos valores normativos nas escalas de exaustão emocional e cinismo bem como pela classificação no terço inferior da distribuição dos resultados normativos na escala de eficácia profissional. A utilização deste critério coloca o problema de não existirem valores normativos estabelecidos para a população portuguesa, correndo-se o risco de considerar "falsos positivos", ou seja, indivíduos que sem apresentarem o problema são considerados como tal. Neste sentido, Shirom (1989) propõe dois critérios de cálculo alternativos, um menos restritivo (valor igual ou superior à frequência "algumas vezes por mês" na escala "Likert") e outro mais conservador (valor igual ou superior à frequência "algumas vezes por semana" na escala "Likert"). No nosso caso, procurámos adoptar um critério intermédio que conciliasse estes dois, assumindose como valor de corte a frequência "uma vez por semana" da escala "Likert" para as dimensões de exaustão emocional e cinismo e "uma vez por mês" para a dimensão de eficácia profissional. Os resultados obtidos apontaram uma percentagem assinalável de profissionais com queixas ao nível da exaustão emocional (11.8\%), seguindo-se a atitude de cinismo face ao trabalho com $9.7 \%$ e a baixa eficácia profissional (7.6\%). Numa análise conjunta da ocorrência de "burnout" nas três dimensões avaliadas pelo instrumento, nenhum dos participantes neste estudo assumiu concomitantemente índices problemáticos nas três facetas, não se podendo assim considerar a existência de profissionais 
com critérios para o fenómeno pleno de "burnout" (apenas quatro casos evidenciaram problemas de exaustão emocional e cinismo simultaneamente).

Quanto aos indicadores de satisfação e realização profissional, alguns dados merecem a nossa atenção. Em primeiro lugar, há que registar o facto de praticamente 1/4 dos participantes $(24.5 \%)$ não voltarem a escolher a mesma saída profissional e, consequentemente, a mesma profissão, se tivessem uma nova oportunidade de optar por uma ocupação laboral. Em termos da satisfação profissional, 7.5\% estão bastante insatisfeitos com a profissão, enquanto $37.6 \%$ estão bastante satisfeitos com a sua actividade (a satisfação moderada é a característica mais comum dos participantes no estudo). Neste mesmo sentido, os valores de realização profissional são bastante baixos para $5.3 \%$ dos militares e bastante altos para 36.2\% (a grande maioria sente-se moderadamente realizada). Já no que respeita aos indicadores relativos ao desejo de abandonar o local de trabalho e a profissão, o principal indicador prende-se com o facto da maioria não pretender sair do posto onde exerce funções $(58.5 \%)$, enquanto $13.8 \%$ manifesta bastante essa intenção. No mesmo sentido, o desejo de deixar a profissão é muito baixo para a esmagadora maioria (71.3\%) e muito alto para uma minoria $(8.5 \%)$.

Por último, as análises das associações e correlações existentes entre todas as variáveis em estudo foram realizadas através do cálculo dos coeficientes de correlação de Pearson, descrevendo-se os valores encontrados na Tabela 1.

Neste caso, aumentos nos níveis globais de "stress" aparecem associados, por um lado, a níveis superiores de exaustão emocional, cinismo e desejo de abandonar o local de emprego e a profissão e, por outro lado, a menores níveis de satisfação/realização (com a vida e com a profissão).

Quanto às dimensões do IBM-VG, constata-se que a exaustão emocional tem uma relação positiva com o cinismo e com a vontade de abandonar local de emprego e profissão e, inversamente, associa-se a uma menor satisfação com a vida e com a profissão. Por sua vez, o cinismo está associado a uma menor satisfação (em todas as dimensões das escalas utilizadas) e a um maior desejo de abandonar o posto de trabalho e o emprego. Quanto à eficácia profissional, pode-se afirmar que maiores níveis nesta dimensão significam igualmente uma maior utilização de "coping" proactivo, comprometimento profissional e satisfação/realização profissional.

Nas restantes dimensões em análise, é de salientar o facto do comprometimento organizacional se associar a maiores níveis de satisfação, em todas as dimensões dos instrumentos utilizados. Como seria de esperar, as duas escalas de satisfação aplicadas neste estudo correlacionam-se positivamente entre si e, por último, os maiores níveis de satisfação/realização correlacionam-se negativamente com o desejo de abandonar o local de emprego e a profissão.

Tabela 1

Média, Desvio-Padrão e Correlações entre as Variáveis em Estudo

\begin{tabular}{|c|c|c|c|c|c|c|c|c|c|c|c|}
\hline VARIÁVEIS & $M$ & $D P$ & 1 & 2 & 3 & 4 & 5 & 6 & 7 & 8 & 9 \\
\hline 1. Nível global de "stress" & 3.52 & .70 & - & & & & & & & & \\
\hline 2. IBM: Exaustão emocional & 1.68 & 1.34 & $.48 * *$ & - & & & & & & & \\
\hline 3. IBM: Cinismo & 1.82 & 1.36 & $.25^{*}$ & $.54 * *$ & 一 & & & & & & \\
\hline 4. IBM: Eficácia profissional & 4.69 & 1.43 & -.12 & .08 & .11 & - & & & & & \\
\hline 5. "Coping" proactivo & 25.81 & 5.07 & -.08 & .15 & .08 & $.43 * *$ & - & & & & \\
\hline 6. Comprometimento organizacional & 30.49 & 6.11 & -.21 & -.18 & -.19 & $.22 *$ & .08 & - & & & \\
\hline 7. Satisfação com a vida & 17.03 & 4.26 & $-.29 * *$ & $-.30 * *$ & $-.35 * *$ & .16 & .09 & $.52 * *$ & - & & \\
\hline 8. Satisfação e realização profissional & 4.15 & .99 & $-.22 *$ & $-.30 * *$ & $-.29 * *$ & $.21 *$ & .04 & $.54 * *$ & $.46 * *$ & - & \\
\hline $\begin{array}{l}\text { 9. Desejo de abandonar local } \\
\text { de emprego e profissão }\end{array}$ & 2.30 & 1.36 & $.22 *$ & $.29 * *$ & $.40 * *$ & -.16 & -.07 & -.19 & -.16 & $.27 * *$ & * - \\
\hline
\end{tabular}

Nota. $* p<.05 ; * * p<.01$.

Variáveis Preditoras da Experiência de "Burnout"

O objectivo destas análises foi compreender quais os factores que melhor poderiam explicar a experiência de "burnout", tomando por base as restantes dimensões avaliadas neste estudo (nível global de "stress", "coping" proactivo, comprometimento organizacional, satisfação com a vida, satisfação/realização profissional, desejo de abandonar o local de emprego e profissão). Neste sentido, efectuamos análises de regressão múltipla, através do método "stepwise".

Assim sendo, no caso da exaustão emocional, o modelo final ficou representado por três variáveis (nível global de "stress", "coping" proactivo e desejo de abandonar o local de emprego e profissão) que, no seu conjunto, explicaram $33 \%$ da variância. Já no cinismo, observamos que $26 \%$ da variância foi explicada pelo desejo de abandonar o local de emprego e a profissão bem como pela satisfação com a vida. Por último, na eficácia profissional foi o "coping" proactivo e o comprometimento que compuseram o modelo final, explicando $21 \%$ da variância. A Tabela 2 fornece indicações mais específicas sobre os valores obtidos nas análises efectuadas.

\section{Diferenças entre Grupos nas Dimensões Avaliadas}

Nesta etapa do estudo procurámos observar se os factores avaliados pelos instrumentos poderiam ajudar a explicar 
Afonso, J. M. P. \& Gomes, A. R. (2009). Stress Ocupacional em Profissionais de Segurança Pública: Um Estudo com Militares da Guarda Nacional Republicana.

Tabela 2

Sumário das Análises de Regressão Múltipla

\begin{tabular}{llllllll}
\hline Variáveis Observadas & Variáveis Preditoras & $R$ & $R^{2}$ & $R^{2 \text { ajust. }}$ & Beta & $t$ & $F$ \\
\hline Exaustão emocional & Nível global "stress" & .484 & .234 & .225 & .454 & 4.88 & $24.48^{* * *}$ \\
& "Coping" proactivo & .539 & .291 & .273 & .258 & 2.81 & $16.19^{* * *}$ \\
& Desejo abandonar local empre./profi. & .594 & .353 & .328 & .256 & 2.75 & $14.20^{* * *}$ \\
Cinismo & Desejo abandonar local empre./profi. & .448 & .201 & .191 & .382 & 3.87 & $20.09^{* * *}$ \\
& Satisfaç. com a vida & .525 & .276 & .258 & -.282 & -2.86 & $15.05^{* * *}$ \\
Eficácia profissional & .437 & .191 & .181 & .419 & 4.23 & $18.88^{* * *}$ \\
& "Coping" proactivo & .481 & .231 & .212 & .202 & 2.04 & $11.89^{* * *}$ \\
\hline
\end{tabular}

Nota. $* * * p<.001$

as diferenças entre subgrupos da nossa amostra, divididos em função de algumas características pessoais e socioprofissionais. Neste sentido, utilizámos análises discriminantes, examinando eventuais diferenças entre subgrupos da nossa amostra em função das pontuações obtidas nas várias dimensões dos instrumentos de avaliação. Para tal, foram constituídos os seguintes grupos de análise: idade, estado civil, nível de escolaridade, experiência profissional e número de horas de trabalho por semana (ver Tabela 3 ). De salientar a nossa preocupação em obter o máximo de equivalência no número de participantes em cada um dos grupos constituídos, de modo a atingir o melhor balanceamento possível nas várias condições observadas. Esta opção jus-tifica o facto de algumas variáveis tipicamente analisadas pela literatura neste tema não estarem incluídas nas aná-lises que se seguem (ex: diferenciação entre homens e mulheres; distinção entre postos profissionais) bem como o facto de não ter sido possível discriminar mais determinados subgrupos (ex: nível de escolaridade e experiência profissional).

Começando pela idade dos militares, constituímos dois grupos distintos que procuraram reflectir as diferenças entre profissionais mais novos (até 40 anos de idade) e mais velhos (mais de 40 anos). Neste caso, encontrámos três variáveis discriminadoras dos grupos, colocando os mais novos a relatar menor desejo de abandonar o local de trabalho e o emprego bem como menor comprometimento organizacional. Inversamente, este grupo evidenciou uma maior tendência para a utilização do "coping" proactivo.

O segundo agrupamento de análise contrastou diferentes estados civis, estabelecendo-se dois grupos de comparação (solteiros e casados). Assim sendo, observou-se maior comprometimento organizacional e vontade em abandonar o local de emprego e a profissão nos militares casados.

No terceiro conjunto de comparações, colocámos em análise diferentes níveis de escolaridade, fixando-se dois grandes grupos: um com níveis de formação mais baixos $\left(1^{\circ} \mathrm{e}\right.$ $2^{\circ}$ ciclos de escolaridade) e outro com um nível mais elevado de ensino (ensino secundário). Neste caso, o comprometimento organizacional e o desejo de abandonar o local de emprego e a profissão são mais prevalentes nos militares com os $1^{\circ}$ e $2^{\circ}$ ciclos, enquanto que o "coping" proactivo é mais utilizado pelos profissionais com o ensino secundário.

No quarto tipo de comparações, definimos dois grupos de comparação de acordo com os anos de experiência profissional nas funções desempenhadas, sendo um característico dos mais inexperientes (até três anos) e outro que tenta representar os militares com maior tempo de serviço (mais de três anos). A este nível, constatou-se que o comprometimento organizacional, o cinismo e o "coping" proactivo foram as variáveis que diferenciaram os grupos, sendo as duas primeiras mais observadas nos militares mais experientes, enquanto a última é mais utilizada pelos seus colegas em início de carreira.

No último conjunto de comparações, colocou-se em análise diferentes horas de trabalho por semana (até 45 horas e mais de 45 horas). Neste caso, verificaram-se duas variáveis discriminadoras, nomeadamente a exaustão emocional e o desejo de abandonar o local de trabalho/emprego, sendo ambas mais prevalentes no grupo com menos horas de trabalho por semana.

\section{Discussão}

O papel das forças policiais nas sociedades ocidentais tem vindo a sofrer uma significativa transformação, reflectindo as mudanças económicas, sociais e tecnológicas nas comunidades bem como a implementação de modelos sociais e políticos de cariz democrático que se baseiam na defesa dos direitos individuais dos cidadãos (Deschamps, Paganon-Badinier, Marchand, \& Merle, 2003). Este tipo de mudanças torna a função de polícia cada vez mais complexa e exigente, sendo fundamental perceber como é que estes profissionais se adaptam aos desafios laborais.

É neste sentido que este trabalho pode ser enquadrado, procurando observar não só a prevalência e frequência de "stress", "burnout" e satisfação, mas também a importância de determinadas variáveis psicológicas, ocupacionais e organizacionais na compreensão desta temática.

Um primeiro aspecto a referir dos resultados obtidos prende-se com o facto das metodologias de avaliação utilizadas terem, de um modo geral, reflectido as estruturas factoriais originais dos instrumentos, sendo estes resultados refor- 
Tabela 3

Diferenças Significativas em Função dos Grupos em Análise: Análise Discriminante

\begin{tabular}{|c|c|c|c|c|c|c|c|c|}
\hline \multirow[t]{2}{*}{ Variável } & \multirow[t]{2}{*}{ "Step" } & \multicolumn{4}{|c|}{ Grupos em análise } & \multirow[t]{2}{*}{ Lambda Wilks } & \multirow[t]{2}{*}{ CEFD (a) } & \multirow[t]{2}{*}{$F$} \\
\hline & & $M$ & $D P$ & $M$ & $D P$ & & & \\
\hline & & \multicolumn{2}{|c|}{ Até 40 anos } & \multicolumn{2}{|c|}{ Mais de 40 anos } & & & \\
\hline "Coping" proactivo & 1 & 27.00 & 4.01 & 24.00 & 5.70 & .911 & .807 & $6.48 *$ \\
\hline Comprome. organizac. & 2 & 29.50 & 5.53 & 32.60 & 6.60 & .831 & .590 & $6.60 * *$ \\
\hline Desejo aband. profiss. & 3 & 2.14 & 1.16 & 2.70 & 1.69 & .773 & -.691 & $6.26 * *$ \\
\hline \multicolumn{9}{|c|}{ Percentagem de participantes correctamente classificados: $72 \%$} \\
\hline & & \multicolumn{2}{|c|}{ Solteiros } & \multicolumn{2}{|c|}{ Casados } & & & \\
\hline Compro. organizacion. & 1 & 28.64 & 5.17 & 31.68 & 6.29 & .941 & .933 & $4.74 *$ \\
\hline Desejo aband. profiss. & 2 & 2.02 & .92 & 2.46 & 1.50 & .889 & .743 & $4.68 *$ \\
\hline \multicolumn{9}{|c|}{ Percentagem de participantes correctamente classificados: $65.9 \%$} \\
\hline & & \multicolumn{2}{|c|}{$1^{\circ}$ e $2^{\circ}$ ciclos } & \multicolumn{2}{|c|}{ Ens. secund. } & & & \\
\hline Compro. organizacion. & 1 & 33.03 & 5.47 & 28.98 & 5.31 & .877 & .906 & $9.96 * *$ \\
\hline Desejo aband. profiss. & 2 & 2.83 & 1.67 & 2.07 & 1.10 & .736 & .746 & $12.55 * * *$ \\
\hline "Coping" proactivo & 3 & 23.62 & 6.07 & 26.86 & 4.13 & .646 & -.598 & $12.59 * * *$ \\
\hline \multicolumn{9}{|c|}{ Percentagem de participantes correctamente classificados: $78.8 \%$} \\
\hline & & \multicolumn{2}{|c|}{ Até 3 anos } & \multicolumn{2}{|c|}{ Mais de 3 anos } & & & \\
\hline Compro. organizacion. & 1 & 28.67 & 5.69 & 32.83 & 5.47 & .875 & .893 & $10.41 * *$ \\
\hline "Coping" proactivo & 2 & 26.87 & 4.38 & 24.58 & 5.77 & .798 & -.665 & $9.09 * * *$ \\
\hline IBM: cinismo & 3 & 1.53 & 1.18 & 2.15 & 1.55 & .712 & .641 & $9.60 * * *$ \\
\hline \multicolumn{9}{|c|}{ Percentagem de participantes correctamente classificados: $75.6 \%$} \\
\hline & & \multicolumn{2}{|c|}{ Até 45 horas } & \multicolumn{2}{|c|}{ Mais de 45 hor. } & & & \\
\hline Desejo aband. profiss. & 1 & 2.91 & 1.39 & 1.91 & 1.22 & .878 & .707 & $10.61 * *$ \\
\hline IMB: exaustão emocio. & 2 & 2.16 & 1.28 & 1.30 & 1.26 & .830 & .579 & $7.71 * *$ \\
\hline
\end{tabular}

Nota. $* \mathrm{p}<.05 ; * * \mathrm{p}<.01$; $* * * \mathrm{p}<.001$; (a) - Coeficiente estandardizado da função discriminante.

çados pelos valores de "alpha" encontrados, que foram bastante aceitáveis.

Adicionalmente, as correlações entre as várias medidas utilizadas apresentaram, na maior parte das variáveis, o "comportamento" esperado. Assim, os maiores níveis de "stress" estão associados a mais problemas nas dimensões do "burnout" (exaustão e cinismo) bem como a um maior desejo de abandonar o emprego e a profissão. De um modo inverso, o nível de "stress" correlacionou-se com menores níveis de satisfação pessoal e profissional. De igual modo, os maiores índices de exaustão emocional e cinismo relacionaram-se com a menor satisfação com a vida e com o trabalho e com o maior desejo de abandonar o emprego e a profissão. Nas restantes variáveis, é de realçar o facto do maior comprometimento organizacional significar maior satisfação com a vida e satisfação/realização profissional. Curioso é o "comportamento" da eficácia profissional do IBM-VG que não se correlacionou com as outras duas dimensões do instrumento. Sem ser esse o objectivo deste trabalho, os dados agora obtidos reforçam as posições avançados por alguns autores, que defendem o facto desta faceta assumir um carácter independente no entendimento do fenómeno do "burnout" (ver Cordes \& Dougherty, 1993; Demerouti, Bakker, Nachreiner, \& Schaufeli, 2001), contrariando-se assim algumas das indicações fornecidas pelos autores do instrumento (Maslach et al., 1996).

No que se refere aos níveis globais de "stress", verificámos neste estudo uma tendência para a maioria dos participantes $(54 \%)$ relatarem níveis significativos a muito elevados de pressão, indo assim de encontro aos dados obtidos por Maslach-Pines e Keinan (2006) com polícias fronteiriços israelitas (52\%) mas situando-se acima dos obtidos por Deschamps et al. (2003) com polícias franceses (33\%). Do ponto de vista da satisfação e realização profissional, os valores encontrados demonstraram alguma variabilidade, salientando-se pelo lado positivo o facto de perto de $40 \%$ dos militares evidenciarem níveis significativos a elevados nestas dimensões. Estes dados são de algum modo corroborados pela circunstância de $75 \%$ dos participantes terem afirmado que voltariam a escolher a mesma profissão se tivessem de voltar a optar por uma actividade laboral (embora não seja de escamotear os $25 \%$ de militares que não voltariam a fazer a mesma escolha...). O desejo de abandonar a profissão e o local de trabalho só é assumida 
por uma minoria de participantes (entre 9 a $14 \%$, respectivamente), enquanto que a maioria manifesta a vontade de se manter na mesma actividade laboral (valores acima dos $50 \%$ ). Também a este nível, é possível encontrar paralelismos com o trabalho de Maslach-Pines e Keinan (2006) onde a maioria dos polícias manifestaram satisfação com a sua profissão (valores acima dos $50 \%$ ). No entanto, tais valores estão claramente abaixo dos encontrados por Deschamps et al. (2003) com indicadores perto dos $90 \%$ (tendo-se curiosamente verificado neste estudo menores níveis globais de "stress").

Quanto aos níveis de "burnout" averiguados neste trabalho, é de salientar o facto de nenhum dos participantes ter assumido problemas em simultâneo nas três dimensões avaliadas pelo IBM-VG, não reunindo critérios para o fenómeno de "burnout" pleno. $\mathrm{Na}$ análise parcial das três facetas do instrumento, a dimensão de exaustão emocional foi a mais prevalente (perto de 12\%), seguindo-se a demonstração de cinismo (quase 10\%) e a baixa eficácia profissional (quase 8\%). Apesar destes índices não serem muito elevados, estão acima dos valores encontrados noutras investigações, nomeadamente em polícias holandeses, onde se concluiu que o fenómeno era praticamente residual (ver Schaufeli \& Buunk, 2003). Paralelamente, o padrão evidenciado pelos militares portugueses também contraria o de outros estudos, onde são as dimensões do cinismo e da falta de realização pessoal que mais se destacam (por contraponto à de exaustão emocional) (ver Kop \& Euwema, 2001).

Numa tentativa de explicar melhor este fenómeno, observamos quais as variáveis preditoras das três dimensões do IBM-VG. Ao nível da exaustão emocional, foi principalmente o nível global de "stress", o "coping" proactivo e o desejo de abandonar o local de emprego e profissão que melhor parecem explicar este problema. O que é curioso observar nesta análise é o facto das estratégias de confronto mais activas também contribuírem para aumentar os sentimentos de exaustão dos militares, parecendo assim assumir-se como uma espécie de "custo associado" à maior tendência destes profissionais em quererem enfrentar e resolver os problemas decorrentes da sua actividade. Na dimensão do cinismo é fundamentalmente a vontade em abandonar o local de emprego e a profissão bem como a menor satisfação com a vida que melhor descrevem esta faceta do esgotamento. Por sua vez, a maior eficácia profissional é predita pelo maior uso do "coping" proactivo e pelos maiores índices de comprometimento organizacional.

O último tipo de análises efectuadas referiu-se às análises discriminantes, que forneceram algumas indicações a ter em consideração. Talvez a mais importante e estruturadora dos resultados obtidos seja a relativa à idade dos militares. Neste caso, verificámos que a maior utilização do "coping" proactivo bem como o menor comprometimento organizacional e desejo de abandonar o local de emprego/profissão caracterizaram os profissionais mais novos. De igual modo os solteiros (e mais novos neste estudo) foram diferenciados dos casados pelo menor comprometimento organizacional e pela menor vontade de abandonar o local de emprego e profissão. No mesmo sentido, os militares com o ensino secundário (e tendencialmente mais novos neste estudo) assumiram menor comprometimento organizacional e desejo de abandonar o local de emprego/ profissão e, inversamente, evidenciaram uma maior orientação para a utilização de estratégias de "coping" proactivas. Ainda na mesma linha de raciocínio, os profissionais menos experientes demonstraram menor comprometimento organizacional e cinismo e, inversamente, maiores níveis de "coping" proactivo. Finalmente, o desejo de abandonar o local de emprego/profissão e a exaustão emocional entraram na análise para discriminar os grupos com diferentes horas de trabalho semanal, observando-se maiores valores nestes domínios nos que cumprem até 45 horas de serviço. Em termos gerais, há duas indicações a reter destes dados: (a) menores níveis de comprometimento organizacional e maior utilização de "coping" proactivo parecem "tipificar" melhor os militares mais novos e/ou inexperientes e (b) maior desejo de abandonar o local de trabalho e a profissão bem como maior cinismo parecem "identificar" melhor os profissionais mais velhos e/ou mais experientes.

Em termos da correspondência destes dados com a literatura é possível estabelecer várias associações, tanto em sentido convergente como divergente. Ao nível da idade e da experiência profissional, os valores verificados, ao colocarem os mais velhos com maior comprometimento organizacional, não confirmam os resultados obtidos por Richardsen, Burke e Martinussen (2006) com polícias noruegueses, onde se averiguou exactamente o contrário, ou seja, foram os profissionais com maior idade que referiram menores níveis de comprometimento.

Do ponto de vista familiar, são vários os estudos que assinalam o impacto negativo do "stress" ocupacional no ambiente familiar dos profissionais casados (Maslach-Pines \& Keinan, 2006; Thompson, Kirk, \& Brown, 2005; Westman \& Etzion, 1995; Williams \& Alliger, 1994) mas, no nosso caso, estes efeitos nocivos fizeram-se sentir mais na carreira profissional (maior desejo de abandonar o emprego e a profissão) e não tanto em termos do sentimento de pertença e comprometimento com a organização (superior nos profissionais casados).

O nível educacional também deverá ser uma variável a ter em consideração neste domínio, uma vez que as diferenças registadas entre profissionais com mais e menos formação escolar reflectiu a importância que lhe é atribuída noutros trabalhos, onde esta variável tende a diferenciar os grupos. A título meramente ilustrativo Burke e Mikkelsen (2006) num estudo com polícias noruegueses, observaram uma tendência dos profissionais com maior nível educacional a relatarem menos queixas físicas e de saúde.

Tendo por base os resultados do nosso estudo, podem ser colocadas duas questões que deverão merecer uma atenção particular da investigação futura. Em primeiro lugar, será interessante saber se a tendência para uma passagem da utilização do "coping" proactivo (caracterizadora dos mais novos) para o cinismo (mais evidente nos mais experientes) identifica o modo como os militares acabam por lidar com os problemas ao longo da sua carreira, "desinteres- 
sando-se" progressivamente das dificuldades e situações que ocorrem à sua volta. Esta questão é tanto mais importante se tivermos em consideração as indicações da literatura que, de um modo sistemático, apontam piores resultados para a saúde e bem-estar dos profissionais na utilização do cinismo e/ou despersonalização face aos outros (ver Bakker \& Heuven, 2006; Burke \& Mikkelsen, 2006). Tal como referem Violanti e Marshall (1983), a utilização do cinismo pelos polícias enquanto estratégia de "coping" (ajudando no distanciamento face aos outros e aos problemas) tem inerente consequências negativas em termos da saúde e do aumento do sentimento de "stress".

Em segundo lugar, pode-se levantar a questão de saber qual a relevância e impacto na cultura organizacional do facto dos mais novos evidenciarem menores níveis de comprometimento face ao local de trabalho. Que factores da estrutura e cultura organizacional poderão estar a promover este facto? Será que esta tendência é algo "natural", resultando do pouco tempo de conhecimento da profissão por parte dos militares mais novos ou traduzirá algum "desencanto" e descontentamento face à profissão? Se esta última hipótese estiver correcta, quais as consequências no desempenho laboral e qualidade dos serviços prestados?

Para terminar, é fundamental que os estudos futuros tenham igualmente em consideração algumas das principais dificuldades e limitações deste trabalho. Desde logo, torna-se indispensável desenvolver metodologias de avaliação do "stress" ocupacional específicas para esta classe profissional (ex: questionários de fontes de "stress") pois só assim será possível compreender os problemas e desafios concretos desta actividade laboral. Por outro lado, a implementação de estudos de natureza qualitativa (ex: entrevistas, "focus group", etc.) poderá ajudar a aprofundar o conhecimento desta temática através de uma maior riqueza na informação recolhida. Finalmente, a implementação de estudos longitudinais também poderá beneficiar a compreensão do modo como os profissionais reagem e se adaptam ao longo do tempo às pressões inerentes da sua profissão, ajudando a clarificar algumas das questões deixadas em aberto nos resultados que obtivemos, tais como, a eficácia e pertinência das estratégias de confronto activas; o impacto do cinismo no individuo e na qualidade do serviço prestado.

\section{Referências}

Abdollahi, A. K. (2002). Understanding police stress research. Journal of Forensic Psychology Practice, 1, 1-24.

Bakker, A. B., \& Heuven, E. (2006). Emotional dissonance, burnout, and in-role performance among nurses and police officers. International Journal of Stress Management, 13, 423440.

Boice, R., \& Myers, P. E. (1987). Which setting is healthier and happier, academe or private practice? Professional Psychology: Research and Practice, 18, 526-529.

Burke, R. J., \& Mikkelsen, A. (2006). Burnout among Norwegian police officers: Potential antecedents and consequences. International Journal of Stress Management, 13, 64-83.
Carlier, I. V. E., Lamberts, R. D., \& Gersons, B. P. R. (2000). The dimensionality of trauma: A multidimensional scaling comparison of police officers with and without posttraumatic stress disorder. Psychiatry Research, 97, 29-39.

Cordes, C., \& Dougherty, T. W. (1993). A review and an integration of research on job burnout. Academy of Management Review, 18, 621-656.

Cruz, J. F., \& Gomes, A. R. (2007). Escala de “Coping” Proactivo $(E C P)$ - Versão para investigação. Manuscrito não-publicado, Universidade do Minho, Braga, Portugal.

Dantzer, M. L. (1987). Police related stress: A critique for future research. Journal of Police and Criminal Psychology, 3, 43-48.

Demerouti, E., Bakker, A. B., Nachreiner, F., \& Schaufeli, W. B. (2001). The job demands: Resources model of burnout. Journal of Applied Psychology, 86, 499-512.

Deschamps, F., Paganon-Badinier, I., Marchand, A. C., \& Merle, C. (2003). Sources and assessment of occupational stress in the police. Journal of Occupational Health, 45, 358-364.

Diener, E., Emmons, R., Larsen, R., \& Griffin, S. (1985). The satisfaction with life scale. Journal of Personality Assessment, 49, 71-75.

Gomes, A. R. (1998). Stress e burnout nos profissionais de psicologia. Dissertação de Mestrado não-publicada, Universidade do Minho, Braga, Portugal.

Gomes, A. R. (2006). Escala de comprometimento organizacional (ECO) - Versão para investigação. Manuscrito não-publicado, Universidade do Minho, Braga, Portugal.

Gomes, A. R. (2007). Inventário de "Burnout" de Maslach (IBM$V G)$ - Versão para investigação. Manuscrito não-publicado, Universidade do Minho, Braga, Portugal.

Gomes, A. R., Melo, B., \& Cruz, J. F. (2000). Estudo do stress e do burnout nos psicólogos portugueses. In J. F. Cruz, A. R. Gomes, \& B. Melo (Eds.), Stress e burnout nos psicólogos portugueses (pp. 73-130). Braga, Portugal: Sistemas Humanos e Organizacionais.

Gorsuch, R. L. (1983). Factor analysis (2nd ed.). Hillsdale, NJ: Erlbaum.

Greenglass, E. R., Schwarzer, R., \& Taubert, S. (1999). The proactive coping inventory (PCI): A multidimensional research instrument. Retrieved April 10, 2007, from http:// www.psych.yourku.ca/greenglass/

Hair, J., Anderson, R., Tatham, R., \& Black, W. (1995). Multivariate data analysis with readings (4th ed.). Upper Saddle, NJ: Prentice-Hall.

Hart, P. M., Wearing, A. J., \& Headey, B. (1995). Police stress and well-being: Integrating personality, coping and daily work experiences. Journal of Occupational and Organizational Psychology, 68, 133-156.

Kop, N., \& Euwema, M. C. (2001). Occupational stress and the use of force by Dutch police officers. Criminal Justice and Behavior, 28, 631-652.

Kyriacou, C., \& Sutcliffe, J. (1978). Teacher stress: Prevalence, sources and symptoms. British Journal of Educational Psychology, 48, 159-167.

Leiter, M. P., \& Durup, J. (1996). Work, home, and in-between: A longitudinal study of spillover. Journal of Applied Behavioral Science, 32, 29-47.

Maslach, C., Jackson, S. E., \& Leiter, M. P. (1996). Maslach Burnout Inventory Manual (3rd ed.). Mountain View, CA: CPP.

Maslach-Pines, A., \& Keinan, G. (2006). Stress and burnout in israeli border police. International Journal of Stress Management, 13, 519-540.

Mowday, R. T., Porter, L. W., \& Steers, R. M. (1982). Employeeorganization linkages: The psychology of commitment, absenteeism, and turnover. New York: Academic Press. 
Mowday, R. T., Steers, R. M., \& Porter, L. W. (1979). The measurement of organizational commitment. Journal of Vocational Behavior, 14, 224-227.

Neto, F. (1993). Satisfaction with life among Portuguese adolescents. Journal of Youth and Adolescence, 22, 125-134.

Neto, F. (1999). Satisfação com a vida e características da personalidade. Psychologica, 22, 55-70.

Nunnally, J. C. (1978). Psychometric theory (2nd ed.). New York: McGraw-Hill.

Richardsen, A. M., Burke, R. J., \& Martinussen, M. (2006). Work and health outcomes among police officers: The mediating role of police cynicism and engagement. International Journal of Stress Management, 13, 555-574.

Rodolfa, E. R., \& Kraft, W. A. (1988). Stressors of professionals and trainees at APA-approved counselling and VA medical center internship sites. Professional Psychology: Research and Practice, 19, 43-49.

Schaufeli, W. B., \& Buunk, B. P. (2003). Burnout: An overview of 25 years of research and theorizing. In M. J. Schabracq, J. A. M. Winnubst, \& C. L. Cooper (Eds.), The handbook of work and health psychology (2nd ed., pp. 383-429). New York: Wiley.

Shaufeli, W. B., \& Enzmann, D. (1998). The burnout companion to study and practice: A critical analysis. London: Taylor \& Francis.

Schaufeli, W. B., Leiter, M. P., Maslach, C., \& Jackson, S. E. (1996). Maslach Burnout Inventory - General Survey (MBIGS). In C. Maslach, S. E. Jackson, \& M. P. Leiter (Eds.), MBI Manual (3rd ed., pp. 19-26). Mountain View, CA: CPP.

Selye, H. (1984). Police stress. Police Stress Magazine, 1, 2-5. Shirom, A. (1989). Burnout in work organizations. In C. L. Cooper \& I. Robertson (Eds.), International review of industrial and organizational psychology (pp. 25-48). New York: Wiley.

Stephens, C., Long, N., \& Flett, R. (1999). Vulnerability to psychological disorder: Previous trauma in police recruits. In J. M. Violanti \& D. Paton (Eds.), Police trauma: Psychological aftermath of civilian combat (pp. 65-74), Springfield, IL: Charles C. Thomas.

Thompson, B. M., Kirk, A., \& Brown, D. F. (2005). Work based support, emotional exhaustion, and spillover of work stress to the family environment: A study of policewomen. Stress and Health, 21, 199-207.

Thoresen, R. W., Miller, M., \& Krauskopf, C. J. (1989). The distressed psychologist: Prevalence and treatment considerations. Professional Psychology: Research and Practice, 20, 153-158.

Violanti, J. M., Andrew, M. E., Burchfiel, C. M., Dorn, J., Hartley, T., \& Miller, D. B. (2006). Posttraumatic stress symptoms and subclinical cardiovascular disease in police officers. International Journal of Stress Management, 13, 541-554.

Violanti, J. M., \& Marshall, J. R. (1983). The police stress process. Journal of Police Science, \& Administration, 11, 389394.

Westman, M., \& Etzion, D. (1995). Crossover of stress, strain and resources from one spouse to another. Journal of Organizational Behavior, 16, 169-181.

Williams, K. J., \& Alliger, G. M. (1994). Role stressors, mood spillover, and perceptions of work-family conflict in employed parents. Academy of Management Journal, 37, 837-868. 